\title{
BMJ Open Active vaccine safety surveillance of seasonal influenza vaccination via a scalable, integrated system in Western Australian pharmacies: a prospective cohort study
}

\author{
Sandra Salter (D) , ${ }^{1}$ Gurkamal Singh, ${ }^{1}$ Lisa Nissen (D) , ${ }^{2}$ Kevin Trentino (D) , ${ }^{3}$ \\ Kevin Murray (D) , ${ }^{3}$ Kenneth Lee (D) , ${ }^{1}$ Benjamin Kop, ${ }^{1}$ Ian Peters, ${ }^{4}$ Alan Leeb ${ }^{4}$
}

To cite: Salter S, Singh G, Nissen L, et al. Active vaccine safety surveillance of seasonal influenza vaccination via a scalable, integrated system in Western Australian pharmacies: a prospective cohort study. BMJ Open 2021;11:e048109. doi:10.1136/ bmjopen-2020-048109

- Prepublication history and supplemental material for this paper is available online. To view these files, please visit the journal online (http://dx.doi org/10.1136/bmjopen-2020048109).

Received 17 December 2020 Accepted 18 May 2021

Check for updates

(C) Author(s) (or their employer(s)) 2021. Re-use permitted under CC BY-NC. No commercial re-use. See rights and permissions. Published by BMJ.

For numbered affiliations see end of article.

Correspondence to

Dr Sandra Salter;

sandra.salter@uwa.edu.au

\section{ABSTRACT}

Objectives We integrated an established participantcentred active vaccine safety surveillance system with a cloud-based pharmacy immunisation-recording program in order to measure adverse events following immunisation (AEFI) reported via the new surveillance system in pharmacies, compared with AEFI reported via an existing surveillance system in non-pharmacy sites (general practice and other clinics).

Design A prospective cohort study.

Participants and setting Individuals $>10$ years receiving influenza immunisations from 22 pharmacies and 90 non-pharmacy (general practice and other clinic) sites between March and October 2020 in Western Australia. Active vaccine safety surveillance was conducted using short message service and smartphone technology, via an opt-out system.

Outcome measures Multivariable logistic regression was used to assess the primary outcome: differences in proportions of AEFI between participants immunised in pharmacies compared with non-pharmacy sites, adjusting for confounders of age, sex and influenza vaccine brand. A subgroup analysis of participants over 65 years was also performed.

Results Of 101440 participants (6992 from pharmacies; 94448 from non-pharmacy sites), 77498 (76.4\%) responded; $96.1 \%(n=74448)$ within 24 hours. Overall, $4.8 \%(n=247)$ pharmacy participants reported any AEFI, compared with $6 \%(n=4356)$ non-pharmacy participants (adjusted OR: 0.87; 95\% Cl: 0.76 to $0.99 ; p=0.039$ ). Similar proportions of AEFls were reported in pharmacy (5.8\%; $n=31)$ and non-pharmacy participants $(6 ; n=1617)$ aged over 65 years (adjusted OR: 0.94; 95\% Cl: 0.65 to $1.35 ; p=0.725)$. The most common AEFls in pharmacy were: pain $(2 \% ; n=104)$, tiredness $(1.9 \% ; n=95)$ and headache $(1.7 \% ; n=88)$; and in non-pharmacy sites: pain $(2.3 \% ; n=1660)$, tiredness $(1.9 \% ; n=1362)$ and swelling $(1.5 \% ; n=1121)$.

Conclusions High and rapid response rates demonstrate good participant engagement with active surveillance in both pharmacy and non-pharmacy participants. Significantly fewer AEFls reported after pharmacist immunisations compared with non-pharmacy

\section{Strengths and limitations of this study}

- We successfully linked two established platforms: an active vaccine safety surveillance system that integrates with national surveillance networks in Australia, with a cloud-based pharmacy immunisation-record system to develop an automated active vaccine safety surveillance system for pharmacies.

- Our response rate was very high, with almost all participants responding within 24 hours.

- Pharmacists are safe immunisers who may capture patients not seen in general practice or other clinics.

- This study provides a proven infrastructure of crucial importance to maintain public safety, to promote confidence in vaccine safety and to assist with vaccine uptake in a safe manner.

- A limitation of our study is that cohort-specific variables such as comorbidities, education or health-seeking behaviours, which could explain the observed differences in adverse events following immunisation proportions, were not measured.

immunisations, with no difference in older adults, may suggest different cohorts attend pharmacy versus nonpharmacy immunisers. The integrated pharmacy system is rapidly scalable across Australia with global potential.

\section{INTRODUCTION}

Pharmacists have an established role in vaccination services. ${ }^{12}$ In Australia, this role has expanded from vaccinating adults with influenza vaccine in the Queensland Pharmacy Immunisation Pilot in $2013,{ }^{3}$ to encompass multiple age groups and vaccines across Australia. Pharmacists in Australia administer vaccines without a prescription to patients in community pharmacies, and in locations outside pharmacies (such as aged care facilities, schools and COVID-19 vaccination hubs). ${ }^{4-6}$ Depending on legislative 
requirements for each state and territory, they can administer influenza vaccines to persons aged 10 years and over; diphtheria-tetanus-acellular pertussis, inactivated poliovirus, measles-mumps-rubella, cholera, Haemophilus influenzae type B, hepatitis A, pneumococcal and meningococcal ACWY vaccines to persons aged $15-16$ years and over; and COVID-19 vaccines to persons aged 18 years and over. ${ }^{5}$ 7-9

Serious reactions to vaccines can and do occur. In 2010, a surge in serious adverse events following immunisation (AEFI) in Western Australian children following vaccination with the seasonal trivalent influenza vaccine led to a Ministerial review, evaluation of the processes for AEFI reporting and investigation of the impact of the 2010 reactions on immunisation rates. ${ }^{10-12}$ This prompted fundamental change in vaccine safety surveillance in Western Australia and Australia. In 2011, the Western Australia (WA) Department of Health launched the (passive) WA Vaccine Safety Surveillance system. ${ }^{13}$ Simultaneously, SmartVax, an automated active surveillance program that employs short message service (SMS) and smartphone technology was developed and implemented in general practice. ${ }^{14}$ AusVaxSafety was also established by the National Centre for Immunisation Research and Surveillance to monitor AEFI with influenza vaccines in children, and now operates as a multicomponent system that includes active vaccine safety surveillance for all vaccines, the Adverse Events Following Immunisation - Clinical Assessment Network, and vaccine safety in primary healthcare data. ${ }^{15-17}$ Importantly, AusVaxSafety uses technology-based surveillance tools, including SmartVax (established in approximately 350 general practice and clinic sites across Australia), to directly contact patients after immunisation in a participant-centred active vaccine safety surveillance approach. ${ }^{15} 18$

This infrastructure precedes pharmacist vaccinations in Australia, yet despite mandated vaccine safety surveillance responsibilities for pharmacists and their planned role in COVD-19 vaccination programmes,${ }^{19-23}$ there are no vaccine safety surveillance tools in use in Australian pharmacies, and no formal systems for post-vaccination follow-up once patients have left the pharmacy. Furthermore, there are no prospective, patient-centred AEFI studies from pharmacy, and no information on differences in AEFI rates between immunisation providers. Therefore it is crucial to link pharmacy with established infrastructure to ensure patients immunised by pharmacists are followed up, and that those AEFI reports are included in national surveillance. A system that uses existing tools, is automated, and linked directly to pharmacy software offers a simple, fail-safe option for surveillance, with little impact on pharmacist workload. MedAdvisor is a global, cloud-based, automated application for pharmacy, ${ }^{24}$ used widely in Australia. The MedAdvisor PlusOne software platform is used by pharmacists to record immunisation encounters, and automatically reports immunisations to the Australian Immunisation Register (AIR). In order to develop a participant-centred, automated active vaccine surveillance system for pharmacies, suitable for monitoring any vaccine delivered by pharmacists, we integrated SmartVax with MedAdvisor. In this pharmacy pilot we sought to: (1). Measure AEFI reported via the integrated pharmacy system, and compare these with AEFI reported via an existing surveillance system in non-pharmacy sites (general practice and other clinics), during the 2020 influenza immunisation season in WA; and (2). Determine the technical success of the integrated pharmacy system via an audit.

\section{METHODS}

\section{Study design, participants and setting}

We conducted a prospective cohort study of participants receiving immunisations from pharmacist and non-pharmacist providers in WA between March and October 2020. Participants were consecutive individuals who self-selected to receive an influenza immunisation at any of 112 locations in WA. This included 22 pharmacies recruited for the purposes of this study and 90 nonpharmacy immunisation sites participating in ongoing active surveillance as part of usual practice.

The aforementioned 22 pharmacies were recruited for SmartVax integration in March 2020 via: direct email to all WA community pharmacies using the MedAdvisor PlusOne software to record immunisations; promotion through professional newsletters and Facebook pages; and via a media release by The University of Western Australia. ${ }^{25}$ Site and pharmacist immuniser consent were obtained prior to enrolling each pharmacy into the study. All pharmacists were experienced in using the MedAdvisor PlusOne software. Training on the SmartVax system and the study was provided to all pharmacists prior to data collection. All pharmacies received printed material to inform participants of the study consent process, with an explanatory poster for display in the immunisation consulting room.

Non-pharmacy sites (including general practice, university, community and local government clinics) participating in active surveillance through SmartVax have been previously described. ${ }^{2627}$

Pharmacists in WA are authorised to administer influenza vaccines to people 10 years and older, so participants aged $\geq 10$ years were eligible for inclusion in this study.

SmartVax was used to actively monitor and record AEFIs between March and October 2020. Immunisations recorded in the MedAdvisor PlusOne software at each pharmacy were automatically batched and sent to SmartVax via a push application programming interface (API) each day. The API was used as a centralised mechanism to submit all records of vaccination encounters recorded across the MedAdvisor cloud application (for participating pharmacies) to the SmartVax server. Immunisation encounters recorded in the non-pharmacy sites were automatically extracted by SmartVax program at each site, each day. 


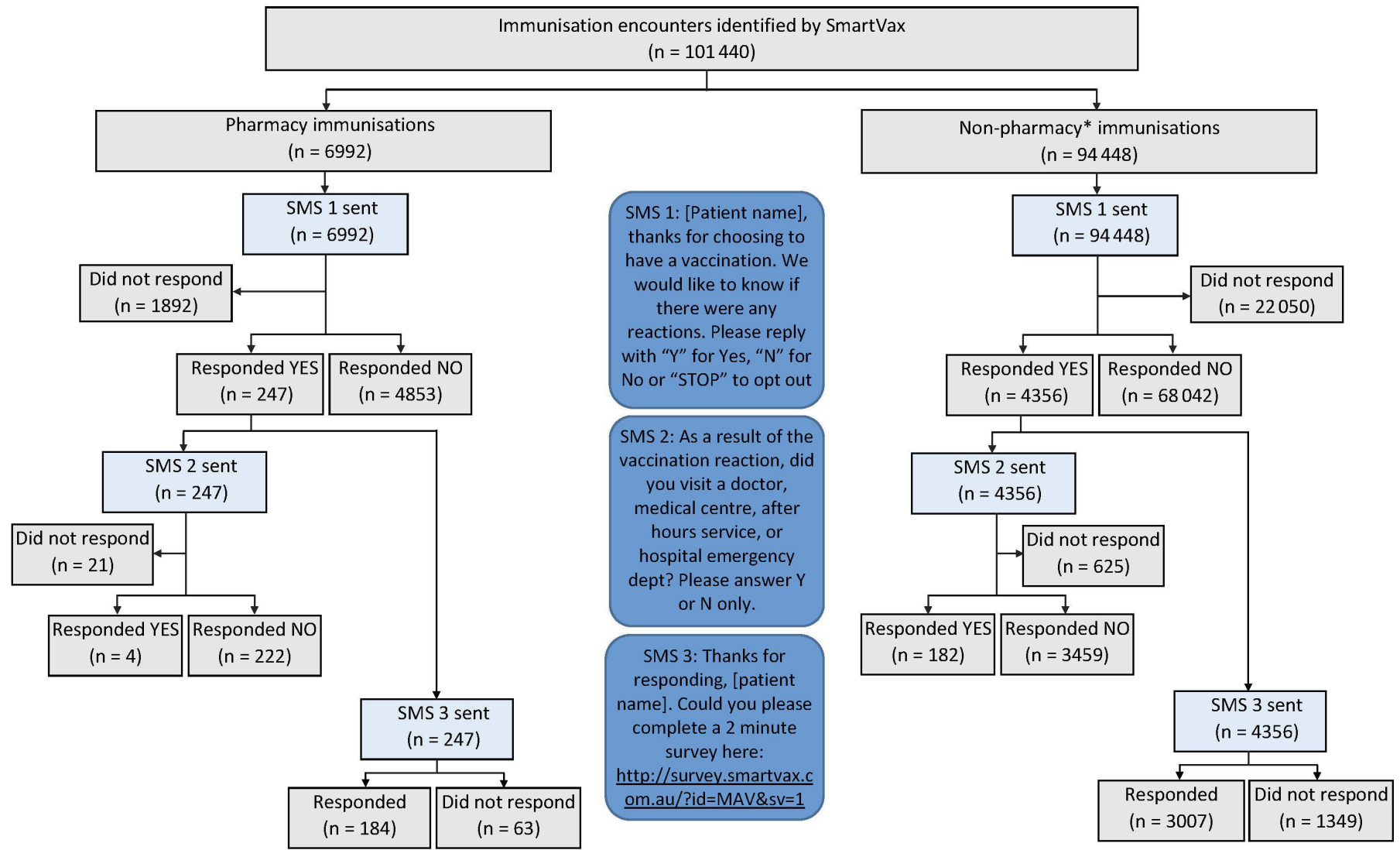

Figure 1 Flow diagram of participant identification for the study cohort. *Non-pharmacy sites included general practices, university clinics, community clinics and local government clinics. SMS, short message service.

SmartVax sent a series of automated SMS text messages to all participants 3-5 days after immunisation, to enquire if any AEFIs had been experienced. In the case of participants $<18$ years of age, SMS messages were sent to the recorded mobile phone number for their parent or legal guardian. 'Yes' responders received a second SMS to enquire whether medical attendance had been sought, followed by a third SMS with a link to a short survey to identify the nature, duration and severity of all reactions (figure 1). The survey enquired specifically about fever, pain, swelling, tired, irritable, sleep, rash, headache, diarrhoea and 'other' AEFI. People who responded 'No' to SMS1 did not receive a second or third SMS.

We also performed an audit of the integrated pharmacy system to determine the number of immunisation encounters recorded in the MedAdvisor PlusOne software that were (1) captured in the batch process, (2) made available for SmartVax surveillance and (3) ultimately underwent surveillance.

\section{Patient and public involvement}

Patients and the public were not involved in designing the integrated pharmacy surveillance system, as we deliberately chose to link pharmacy with broader vaccine surveillance infrastructure, which by nature requires a uniform structure and is unable to be changed. Patients and the public were not involved in the conduct, reporting or dissemination of results.

\section{Variables}

The primary outcome of interest was any AEFI. Secondary outcomes included AEFIs resulting in medical attendance, and adverse event profile as reported in the survey (fever, pain, swelling, tired, irritable, sleep, rash, headache, vomiting, diarrhoea and other). In addition to data collected on adverse events, SmartVax captured information on participant age, sex and influenza vaccine brand. In order to compare adverse events reported by participants immunised in pharmacies to non-pharmacy sites, our exposure variable of interest was immunisation provider.

Data for the analysis were obtained from the SmartVax system, which includes participant demographic data, their responses to SMS messages, and where relevant, survey results.

\section{Statistical methods}

Summary statistics for each immunisation location were provided and comparisons between pharmacy and nonpharmacy immunisations were made using the independent samples t-test (or Mann-Whitney test for non-normal data) for continuous variables and $\chi^{2}$ tests for categorical variables. A comparison of participants reporting adverse events following influenza immunisation was made in unadjusted analysis using a $\chi^{2}$ test. Logistic regression was used to assess differences in proportions of adverse events between participants immunised in pharmacies 
and participants immunised at non-pharmacy sites, adjusting for confounders of age (as a continuous variable), sex and influenza vaccine brand in a multivariable model. People aged 65 years and over are at higher risk of complications from influenza and are recommended to receive an adjuvanted influenza vaccine. In 2020, a new, quadrivalent vaccine (Fluad Quad (Seqirus)), was used for the first time in the Australian population, therefore we conducted a subgroup analysis of participants in this age group. Only complete records (age, sex and influenza vaccine brand) were included in the analyses. The significance level was set at 0.05 .

All statistical analyses were conducted using R (V.3.5.3; The R Foundation for Statistical Computing). Results are reported according to Strengthening the Reporting of Observational Studies in Epidemiology checklist for cohort studies. $^{28}$

\section{RESULTS}

A total 101849 influenza immunisation encounters were eligible for surveillance between 10 March 2020 and 17 October 2020. Of these, 409 were missing sex, leaving 101440 complete influenza immunisation encounters for active surveillance (6992 from pharmacies and 94448 from non-pharmacy sites). A total 5100 $(72.9 \%)$ pharmacy participants and 72398 (76.7\%) non-pharmacy participants responded to SMS1, providing 77498 immunisation encounters for analysis (figure 1).

The mean (SD) age of participants was 51.5 (21.02) years and $58.3 \% \quad(\mathrm{n}=45186)$ were female. The most common influenza vaccines administered were FluQuadri (Sanofi-Aventis): $27.1 \% \quad(\mathrm{n}=27491)$, Fluad Quad (Seqirus): $26.6 \% \quad(\mathrm{n}=26934)$ and Afluria Quad (Seqirus): 10.3\% ( $\mathrm{n}=10491)$. Overall, 96.1\% ( $\mathrm{n}=74448)$ of responders replied within 24 hours of receiving SMS1, with $98.7 \%(\mathrm{n}=76485)$ responding within 72 hours. Of all responders, $5.9 \%(\mathrm{n}=4603)$ reported an adverse event following immunisation.

We present the characteristics of participants immunised in table 1 .

Participants receiving influenza vaccinations at pharmacies had a lower mean age (46.5 vs 51.9 years; $\mathrm{p}<0.001)$, with $10.5 \%(\mathrm{n}=533)$ aged 65 years and over compared with $37.4 \% \quad(\mathrm{n}=27042)$ at non-pharmacy sites. Pharmacists were more likely to administer Afluria Quad (Seqirus) and Fluarix Tetra (GlaxoSmithKline), while non-pharmacist immunisers were more likely to administer Fluad Quad (Seqirus) and FluQuadri (SanofiAventis) $(\mathrm{p}<0.001)$. Response times to the first SMS were similar between groups, with a median (IQR) of $13 \mathrm{~min}$ (3-72 min) for pharmacy and $14 \mathrm{~min}(4-70 \mathrm{~min})$ for nonpharmacy vaccinees.

Table 1 Characteristics of influenza immunisation encounters by location

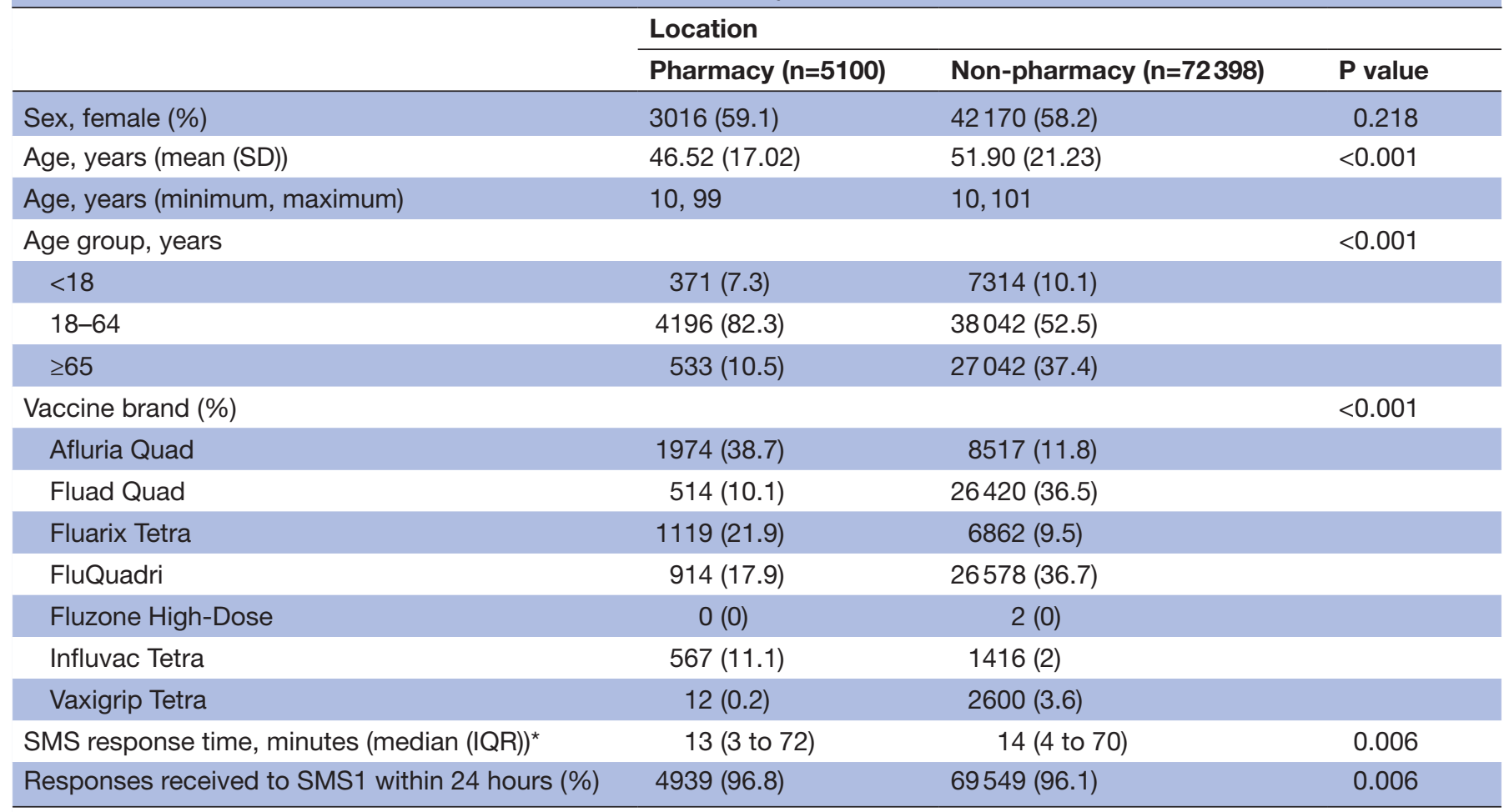

Values are count (proportion), mean (SD) or median (IQR) as indicated. P values are calculated from the $\chi^{2}$ test for categorical variables and the independent samples t-test for continuous variables.

${ }^{*}$ The $\mathrm{p}$ value for SMS response time was calculated using the Mann-Whitney Wilcoxon Test.

SMS, short message service. 


\section{Rate of any adverse event}

The unadjusted proportion of any adverse event differed significantly between participants immunised at pharmacies and those immunised elsewhere. Of those immunised at pharmacies, $4.8 \%(n=247)$ reported an adverse event, compared with $6 \%(n=4356)$ for those immunised at nonpharmacy sites (unadjusted $\mathrm{OR}=0.80 ; 95 \%$ CI: 0.70 to 0.91 ; $\mathrm{p}=0.001)$. After adjusting for age, sex and vaccine brand, participants immunised at pharmacies reported fewer adverse events, compared with participants immunised in non-pharmacy sites ( $\mathrm{OR}=0.87 ; 95 \% \mathrm{CI}$ : 0.76 to 0.99 ; $\mathrm{p}=0.039)$.

\section{Adverse events resulting in medical attendance}

Participants reporting any adverse event were sent a second SMS asking if they visited a doctor, medical centre, after-hours service or hospital emergency department as a result of their vaccination reaction. We excluded 646 $(0.8 \%)$ encounters from this analysis, as they did not respond to the second SMS.

Of participants immunised at pharmacies, $0.1 \% \quad(n=4)$ reported seeking medical care following an adverse event compared with $0.3 \%(\mathrm{n}=182)$ of participants immunised at non-pharmacy sites $(\mathrm{p}=0.021)$. After adjusting for age, sex and vaccine brand, participants immunised at pharmacies reported fewer adverse events for which medical attendance was sought, compared with participants immunised at non-pharmacy sites ( $\mathrm{OR}=0.35 ; 95 \%$ CI: 0.13 to $0.97 ; \mathrm{p}=0.042)$.

\section{Adverse event profile}

The most common adverse events reported after immunisation in pharmacies were pain $(2 \% ; \mathrm{n}=104)$, tiredness $(1.9 \% ; \mathrm{n}=95)$ and headache $(1.7 \% ; \mathrm{n}=88)$. The most common adverse events reported after immunisation in non-pharmacy sites were pain $(2.3 \% ; n=1660)$, tiredness $(1.9 \% ; \mathrm{n}=1362)$ and swelling $(1.5 \% ; \mathrm{n}=1121)$ (figure 2$)$. A total of $1.4 \% \quad(n=68)$ pharmacy and $1.2 \% \quad(n=778)$ non-pharmacy participants selected 'other' AEFI, and included a text description of the reaction. The majority of these reactions were described as aches, dizziness, muscle and joint pain, cold symptoms, sore throat and nausea (online supplemental tables 1 and 2). Figure 3 presents ORs for adverse event profile after adjusting for age, sex and vaccine brand, comparing participants immunised at pharmacies to those immunised at nonpharmacy sites.

\section{5 years and over subanalysis}

Of participants aged 65 years and over, $5.8 \% \quad(n=31)$ immunised at pharmacies reported any adverse event compared with $6 \%(\mathrm{n}=1617)$ for those immunised at nonpharmacy sites (OR=0.97; 95\% CI: 0.67 to $1.40 ; \mathrm{p}=0.875)$. After adjusting for age, sex and vaccine brand, participants immunised at pharmacies reported similar proportions of any adverse events when compared with participants immunised at non-pharmacy sites ( $\mathrm{OR}=0.94$; $95 \%$ CI: 0.65 to $1.35 ; \mathrm{p}=0.725)$. No participants 65 years and over who were immunised at pharmacies reported seeking medical care following an adverse event, compared with $0.3 \%$ $(n=72)$ of participants immunised at non-pharmacy sites.

\section{Audit of the integrated pharmacy system}

Of 11008 immunisations given in the pharmacies, $10 \%$ $(\mathrm{n}=1106)$ chose not to participate in surveillance, and $71 \%(\mathrm{n}=7821)$ were captured in the API batch process. Of these, $95.6 \% \quad(n=7475)$ were available for SmartVax surveillance and $92.4 \%(n=7230)$ were sent SMS1. We identified in July 2020 that $20.1 \%(n=2081)$ records for participants without a recorded sex had not transferred via the API. This was rectified and subsequently $100 \%$ of records (including records without sex) were successfully transferred via the API (figure 4).

\section{DISCUSSION}

Active surveillance systems, particularly those that integrate new technologies with current vaccine surveillance systems, ${ }^{29}{ }^{30}$ are vital to maintain public health and confidence in vaccination, and are directly applicable to the current global vaccination campaign against COVID-19. To the best of our knowledge, this study is the first to

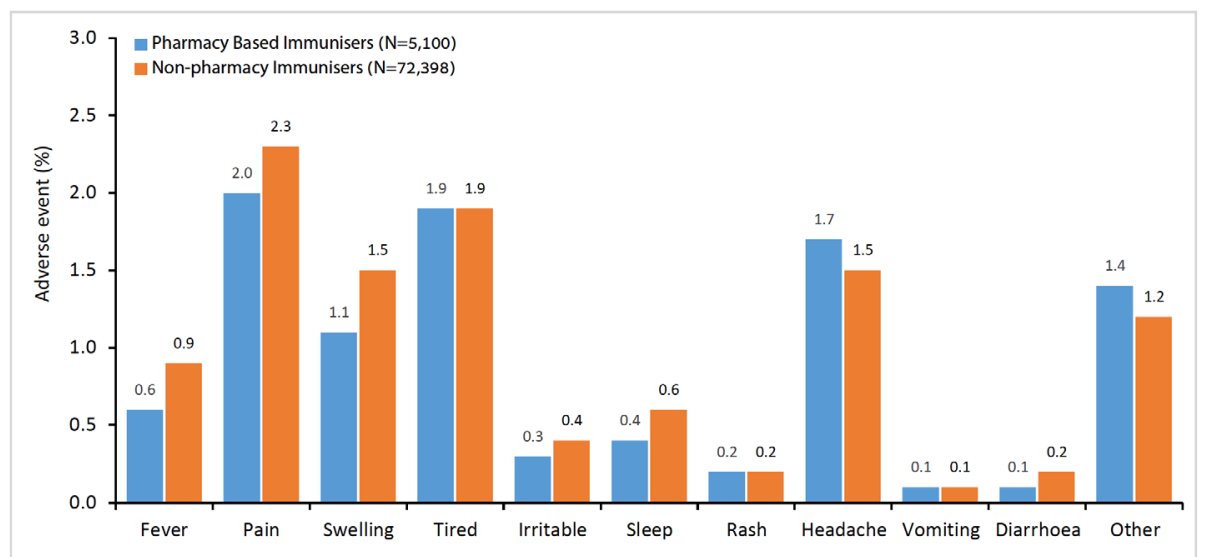

Figure 2 Unadjusted proportion of adverse events (\%) reported following immunisation, stratified by provider. 


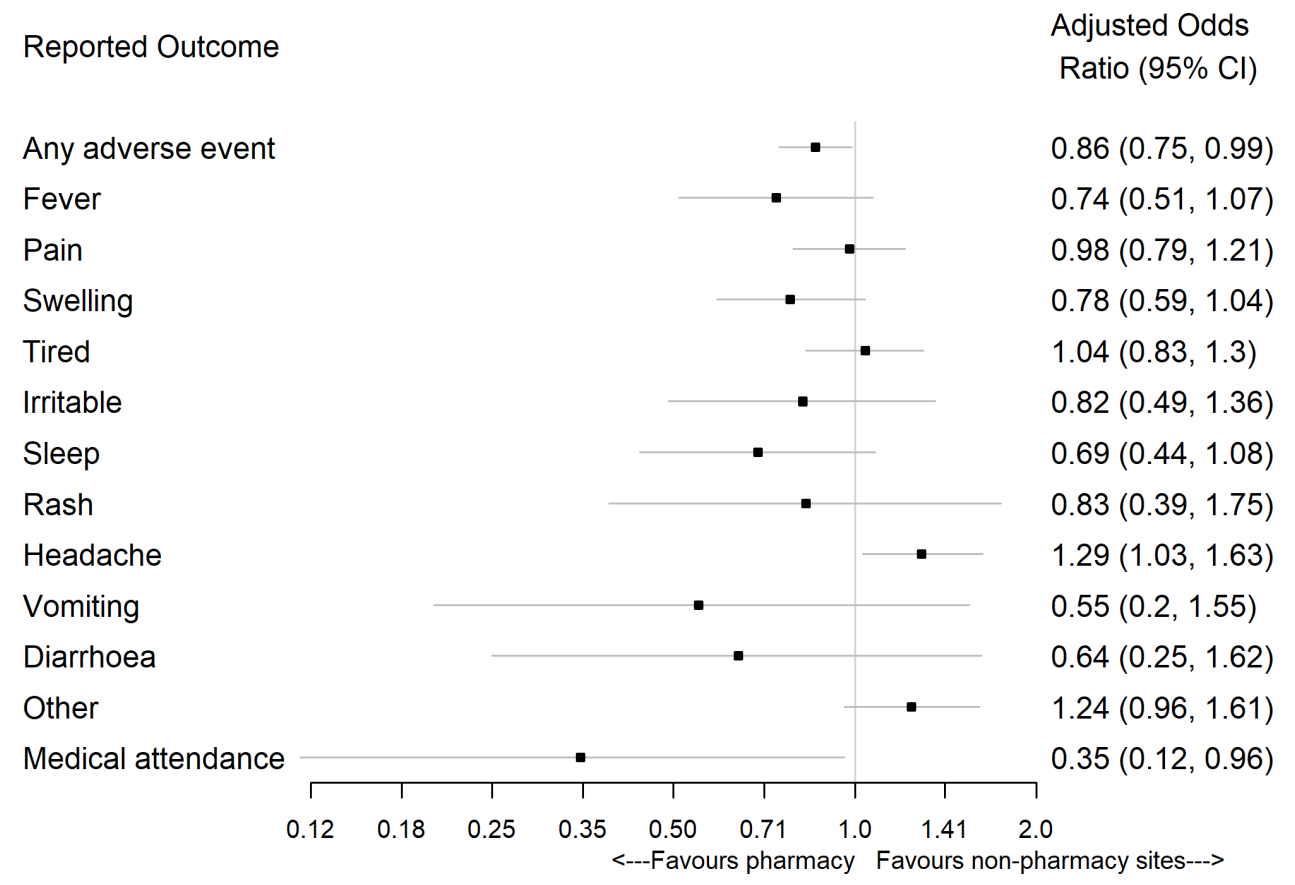

Figure 3 Adjusted ORs $(95 \% \mathrm{Cl})$ comparing proportion of adverse events reported following immunisation in pharmacies to non-pharmacy sites.

implement and evaluate a system for active surveillance of AEFI in pharmacies.

Through integration of established active vaccine surveillance and cloud-based pharmacy software systems, we developed a robust, rapidly scalable, automated, user-friendly, large scale AEFI monitoring system. While both pharmacy and non-pharmacy sites used the same SmartVax tool to monitor AEFI, only pharmacies made use of the integrated system: non-pharmacy sites use SmartVax as a locally installed software program, whereas the integrated system used cloud technology, and therefore has the advantage of scalability. There was a high $(73 \%)$ and rapid response to active surveillance following influenza immunisation in pharmacies (median $13 \mathrm{~min}$; 96\% within 24 hours); similar to responses observed in non-pharmacy sites, both in this study and previously. ${ }^{26}{ }^{27}$ While the profile of adverse events reported after pharmacy-based immunisations was similar to that reported after immunisation in non-pharmacy sites, there were significantly fewer adverse events overall reported from participants immunised in pharmacies compared with those immunised in non-pharmacy sites, including general practice and other clinics. After adjusting for age, sex and vaccine brand this difference was still significant, although clinically small. As participants immunised in pharmacies were significantly younger than those in nonpharmacy sites, it is possible that more complex or sicker participants visited non-pharmacy immunisers. Further our analysis could not be adjusted for underlying comorbidities or social factors such as income (which could influence ability to pay for vaccination in the pharmacy). However, there was no difference in overall adverse events observed in our subanalysis of the over 65 years age group, whom we might expect to have more comorbidities or be sicker. Notably, a surge in demand for early influenza vaccination in 2020, means we did not capture all participants immunised in participating pharmacies, as our integrated system was activated a month after pharmacy vaccinations began. This could have included sicker patients of any age, as they may seek immunisation as early as possible in the season. The relationship with the immuniser, anticipation of AEFI or sense of obligation to report even minor reactions may be different for people attending pharmacies and non-pharmacy sites, but we did not measure this. Furthermore, each site can and does immunise patients opportunistically. Such immunisation may, by default, affect the cohort attending and their perception of AEFIs: people opportunistically immunised in non-pharmacy sites (including general practice) may perceive themselves to be sicker or may feel obliged to report AEFIs to their doctor, although we did not measure the proportion of booked versus opportunistic immunisations, or ask patients directly about their perceptions. Regardless, given response rates and time to respond were similar between groups, the reason for the observed difference and whether it is a true effect remains unclear. Even if the difference is true, it may simply represent different cohorts being immunised in different sites: this in itself is of benefit as it indicates pharmacies may capture a different set of people who may otherwise not seek immunisation. Importantly, engagement with the integrated pharmacy system was observed in 5100 participants (aged 10-99 years) across 22 pharmacies, illustrating that age was not a barrier to pharmacy vaccination, nor the technology used in the system.

This study has the potential to change practice immediately. Most pharmacy vaccinations in Australia are recorded using two cloud-based software platforms, each 


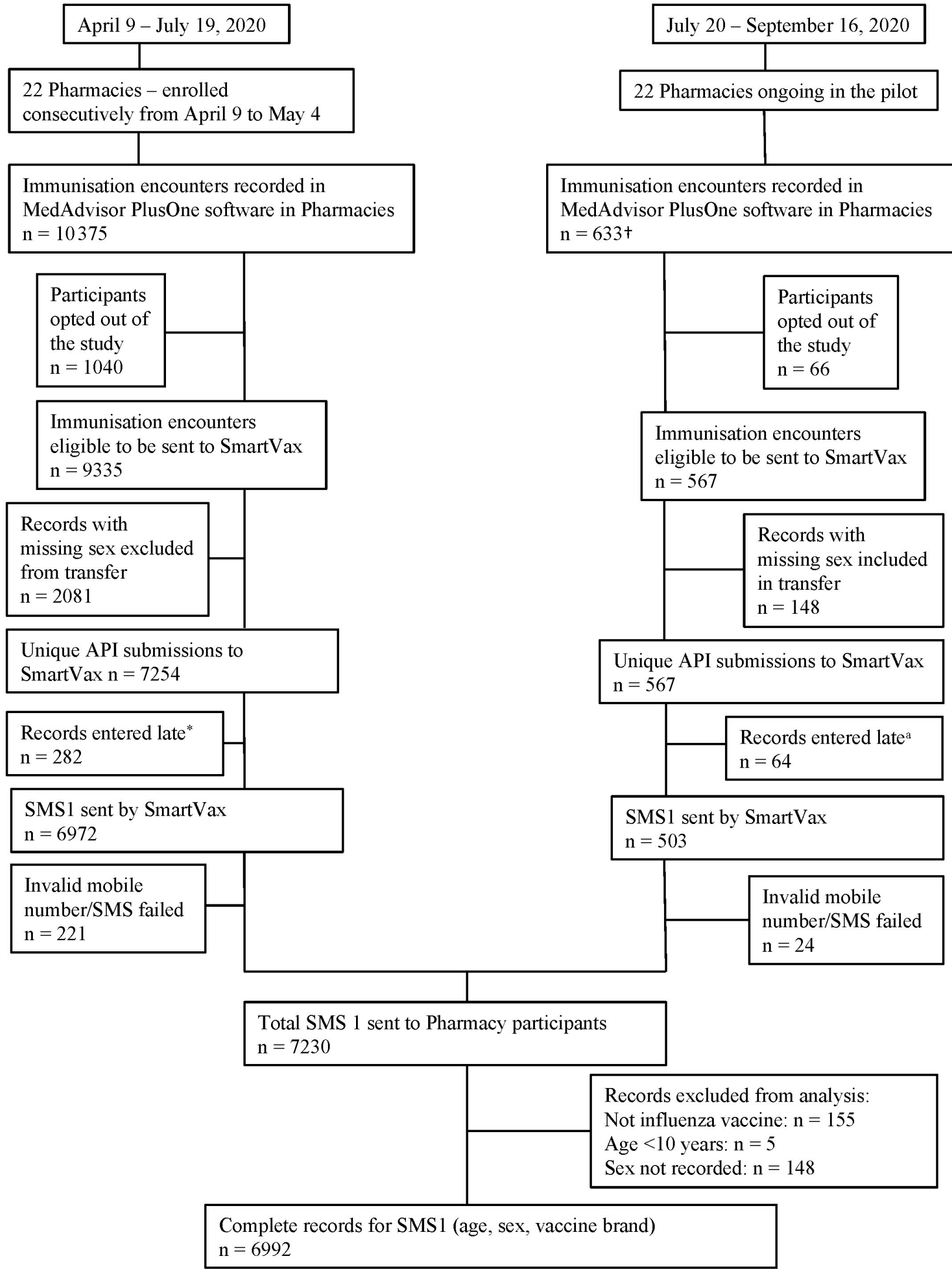

Figure 4 Audit of data flow in the integrated pharmacy active vaccine safety surveillance system. *SmartVax automatically monitors all encounters three business days after immunisation. Therefore encounters entered to MedAdvisor PlusOne more than three business days after immunisation did not receive SMS1. This scenario occurred in pharmacies with a paper-toelectronic recording system. These encounters still transferred via the API but did not meet criteria for automatic surveillance. †Unseasonably high demand for influenza vaccination early in the season resulted in low numbers of immunisations after July. API, application programming interface; SMS, short message service. 
of which automatically reports to the AIR. ${ }^{31}$ Previously, there were no active vaccine safety surveillance systems and no mechanism to identify AEFI once patients left the pharmacy. Through this study, active vaccine safety surveillance is now scalable to thousands of pharmacies in Australia and can monitor patients out of reach of other surveillance networks. Furthermore, there is potential for global application given the cloud-based pharmacy software integrated in this study, MedAdvisor, has an international presence. This may be particularly useful for pharmacies in countries where there does not appear to be published evidence of ongoing active surveillance systems, for example, the UK. ${ }^{32}$

Significant economies of scale are achieved through the pharmacy integration: technical support (including program updates and modifications to surveillance parameters), or addition of surveillance fields (such as medication lists or demographic variables), can be achieved efficiently in either component of the integrated system and activated in real-time across participating sites. This could offer an advantage over non-pharmacy systems, which currently use locally, installed software. The integrated system can also contribute to research, such as to provide long-term vaccine surveillance data for linkage to national or state based administrative data sets (eg, to enable comparison of actively reported AEFI against long-term outcomes or infection). Connection through the integration to AusVaxSafety, the principal Australian AEFI active surveillance system, means pharmacy AEFI can be included in publicly reported surveillance data, ensuring open and transparent monitoring. In context of high social media-led disinformation campaigns about vaccine safety, ${ }^{33}$ open reporting promotes public confidence in the vaccine and the surveillance system, and can mitigate anti-vaccination campaigns. ${ }^{12}$ While provider recommendations drive immunisation, ${ }^{34}$ public and provider belief that regulatory systems are robust remain critical to uptake and high immunisation rates required for herd immunity. ${ }^{35}$

Rapid engagement of a trained immunisation workforce with access to active vaccine safety surveillance will be crucial for COVID-19 vaccine deployment. Pharmacists have been recognised as qualified immunisers in many countries for more than 10 years. ${ }^{2}$ Two systematic reviews have demonstrated pharmacists increase vaccination coverage when compared with traditional (non-pharmacist) providers alone. ${ }^{36}$ Several factors specific to pharmacies including convenience, accessibility, extended opening hours and the ability to obtain a vaccination without prior appointment have been shown to increase immunisation rates across the world and it is likely pharmacies capture patients who would otherwise not get immunised. ${ }^{123637}$ In the Australian context, cost is an interesting factor. Most pharmacy vaccination services are not remunerated by Australian state or federal governments and patients pay the full cost for the vaccine as well as any service fees. By contrast, vaccination service fees in general practice and non-pharmacy clinics are subsidised either partially or fully by the federal government, and vaccines are provided free for all eligible patients under the National Immunisation Program (NIP), including for babies, children and adults. Since 2019, a government programme to expand access to influenza vaccines has enabled participating pharmacies in WA and the Australian Capital Territory to administer influenza vaccinations to persons aged 65 years and older, under NIP. Pharmacies in this programme receive free vaccine but no service fee, and may choose to charge a service fee to the patient, ${ }^{7}$ which means for the patients of those pharmacies aged over 65 , it is still more expensive to be vaccinated in the pharmacy than a non-pharmacy site. Not all pharmacies in our study participate in the NIP programme, and we did not distinguish patients aged over 65 in the pharmacy subgroup who may have been eligible for an NIP funded vaccination, as this detail was not available.

A key strength of this study was our technical audit, to confirm immunisation records transferred as expected using the integrated pharmacy system. To the best of our knowledge this is the only audit of data flow in an active surveillance system. We identified three key issues. First, the API did not identify records without sex recorded, and as a result, $21 \%$ of pharmacy immunisations were not automatically monitored in the first half of this study. This issue was identified, the system was updated and all records subsequently transferred. Future iterations of the integrated system should enforce recording of all patient data during the consent process. Second, a small proportion of pharmacists used a paper-based system to record vaccination encounters, and subsequently transposed those records to the electronic system. This may have been a stop-gap measure during the unprecedented demand period of early COVID-19 activity, or it may represent usual practice. Records transferred more than three business days after immunisation were not automatically monitored, and the reason for the paper system was not elucidated. Nonetheless, this represents an area where pharmacists must improve their practice: from 2021 it is mandatory for all vaccination providers to report vaccines given to the AIR, ${ }^{38}$ and timely record keeping is essential for this. Third, 3\% of participants did not have a valid mobile phone number recorded and were not monitored. Attention should be given to identify whether participants own a mobile phone, and if so, to accuracy in data entry. Access to technology may also pose an issue for a wider population as the integrated pharmacy system requires internet connectivity for the pharmacist and mobile network connectivity for the patient. Beyond the audit, approximately $10 \%$ of pharmacy participants declined to participate in the study and were not sent SMS1. This may have been a feature of not wishing to participate in a research project, rather than wishing to avoid monitoring. It is not possible to predict whether participants would choose to participate in monitoring under real-world conditions, or whether different vaccines could affect interest in receiving monitoring. 
While the integrated pharmacy system provides de-identified AEFI data (collected using the SmartVax tool) to AusVaxSafety for the purposes of signal detection, it was not designed to deliver identified AEFI patient data back to the pharmacist. It is essential that serious AEFI are reported back (as identified data) to the pharmacist, so they can follow-up with the patient and undertake reporting-which is mandatory in some states and territories in Australia-to local health authorities for individual patient investigation. This is particularly important for COVID-19 vaccines where AEFI rates are unknown and may be higher than for seasonal influenza vaccines, and where patients could receive each of their two doses of vaccine across different sites. Notably, in Australia, active vaccine safety surveillance is usually conducted 3 days after vaccination, whereas COVID-19 vaccines will be monitored on days 3,8 and $42 .{ }^{15}$ Should serious reactions occur at any of those time points, the vaccinator needs to know, before dose two is provided. Further urgent work with the integrated system is required to complete this step. Beyond this, it is important to consider the utility, workload and benefits or otherwise of the integrated system, and future research should be directed to evaluate pharmacists' perceptions of and experiences with this system.

\section{CONCLUSION}

We are on the cusp of a new period in immunisation. The crucial next step is to leverage proven technology to enable broad scale deployment of vaccines, with a trained workforce enabled with vaccine safety surveillance systems. We have developed an integrated active vaccine safety surveillance system that is scalable to thousands of pharmacies in Australia and potentially globally. Lower proportions of adverse events following influenza immunisation in pharmacies compared with non-pharmacy sites demonstrates pharmacies are a safe destination for immunisation, and may capture people who might otherwise not get vaccinated. With an integrated system that facilitates both reporting of the immunisation to the AIR, as well as links to national vaccine safety surveillance, pharmacists in Australia can contribute actively and safely to all immunisation programmes.

\section{Author affiliations \\ ${ }^{1}$ Division of Pharmacy, The University of Western Australia School of Allied Health, Perth, Western Australia, Australia \\ ${ }^{2}$ Clinical Sciences, Pharmacology and Pharmaceutical Sciences, Queensland University of Technology, Brisbane, Queensland, Australia \\ ${ }^{3}$ The University of Western Australia School of Population and Global Health, Perth, Western Australia, Australia \\ ${ }^{4}$ Illawarra Medical Centre, Perth, Western Australia, Australia}

Twitter Sandra Salter @SandraMSalter and Kevin Trentino @kevintrentino

Acknowledgements We thank Ms Dani Li, Mr Craig Schnuriger, Mr Weyn Ong, Mr Daniel Smith, Ms Karin Orlemann, Ms Danae Perry, Ms Rachel Nesaraj and Mr Nicolas Bchara for their continued efforts and contributions. We thank SmartVax and MedAdvisor International for their support and dedication to the project.
Contributors SS conceptualised, designed and supervised the study, raised funding for the study, assisted with data collection and analysis, interpreted the results, wrote the draft versions and prepared the final version of the manuscript. GS performed the literature review, assisted with data collection and interpretation of the results and reviewed the manuscript. LN raised funding for the study, assisted with study design and recruitment, interpreted the results and reviewed the manuscript. KT assisted with data collection, verified the underlying data, performed data analysis, interpreted the results, prepared all charts, wrote part of the draft versions and reviewed the manuscript. KM assisted with data collection, verified the underlying data, assisted with data analysis, interpreted the results and reviewed the manuscript. KL assisted with study design, raised funding and reviewed the manuscript. BK performed the literature review, assisted with data collection and interpretation of the results and reviewed the manuscript. IP provided intellectual and technical input, provided access to active surveillance, assisted with study supervision, data collection and analysis, interpreted the results and reviewed the manuscript. AL provided intellectual input, provided access to active surveillance, assisted with study supervision, data collection and analysis, interpreted the results and reviewed the manuscript. All authors have read and approved the final version of the manuscript.

Funding The research was supported by a research grant provided by the $\mathrm{J} M$ 0'Hara Research Fund of the Pharmaceutical Society of Western Australia. Grant number not applicable.

Competing interests AL and IP are Directors of SmartX Data, of which SmartVax is a subsidiary. IP has previously received funding from SmartX Data for database development separate to this study.

Patient consent for publication Not required.

Ethics approval Approval to conduct this research was obtained from the Human Research Ethics Committee of The University of Western Australia.

Provenance and peer review Not commissioned; externally peer reviewed.

Data availability statement Data are available upon reasonable request. Individual participant data that underlie the results reported in this article, after de-identification (text, tables, figures and supplemental material) are available upon reasonable request. Proposals for data should be directed to Sandra Salter via ORCID: https://orcid.org/0000-0002-5840-6797. To gain access to data, users will need to sign a data access agreement. Reuse is permitted under the following conditions: meta-analyses of adverse events following immunisation in pharmacies.

Supplemental material This content has been supplied by the author(s). It has not been vetted by BMJ Publishing Group Limited (BMJ) and may not have been peer-reviewed. Any opinions or recommendations discussed are solely those of the author(s) and are not endorsed by BMJ. BMJ disclaims all liability and responsibility arising from any reliance placed on the content. Where the content includes any translated material, BMJ does not warrant the accuracy and reliability of the translations (including but not limited to local regulations, clinical guidelines, terminology, drug names and drug dosages), and is not responsible for any error and/or omissions arising from translation and adaptation or otherwise.

Open access This is an open access article distributed in accordance with the Creative Commons Attribution Non Commercial (CC BY-NC 4.0) license, which permits others to distribute, remix, adapt, build upon this work non-commercially, and license their derivative works on different terms, provided the original work is properly cited, appropriate credit is given, any changes made indicated, and the use is non-commercial. See: http://creativecommons.org/licenses/by-nc/4.0/.

\section{ORCID iDs}

Sandra Salter http://orcid.org/0000-0002-5840-6797

Lisa Nissen http://orcid.org/0000-0001-5826-4605

Kevin Trentino http://orcid.org/0000-0002-8768-9263

Kevin Murray http://orcid.org/0000-0002-8856-6046

Kenneth Lee http://orcid.org/0000-0003-3022-4868

\section{REFERENCES}

1 Burt S, Hattingh L, Czarniak P. Evaluation of patient satisfaction and experience towards pharmacist-administered vaccination services in Western Australia. Int J Clin Pharm 2018;40:1519-27.

2 Poudel A, Lau ETL, Deldot M, et al. Pharmacist role in vaccination: evidence and challenges. Vaccine 2019;37:5939-45.

3 Nissen LM, Glass BD, ETL L. Queensland pharmacist immunisation pilot (QPIP) phase 1 pharmacist vaccination-influenza final report. 
Brisbane: Queensland University of Technology, 2015. https://eprints. qut.edu.au/91903/

4 International Federation of Pharmacy. An overview of current pharmacy impact on immunisation: a global report. Available: https:// www.fip.org/files/fip/publications/FIP_report_on_Immunisation.pdf [Accessed 02 Dec 2020].

5 Pharmaceutical Society of Australia. COVID-19 information for pharmacists, 2021. Available: https://www.psa.org.au/coronavirus/\# 1613955340819-3ab219ba-bdc1 [Accessed 31 Mar 2021].

6 McDerby NC, Kosari S, Bail KS, et al. Pharmacist-Led influenza vaccination services in residential aged care homes: a pilot study. Australas J Ageing 2019;38:132-5.

7 The Pharmacy Guild of Australia. Vaccination services, 2021. Available: https://www.guild.org.au/programs/vaccination-services [Accessed 31 Mar 2021].

8 Paola S. New year, new pharmacist vaccines. Aust J Pharm, 2019. Available: https://ajp.com.au/news/new-year-new-pharmacistvaccines/

9 Scholefield A. Pharmacists granted sweeping new vaccination role. Australian Doctor News, 2017. Available: https://www.ausdoc.com. au/news/pharmacists-granted-sweeping-new-vaccination-role

10 Armstrong PK, Dowse GK, Effler PV, et al. Epidemiological study of severe febrile reactions in young children in Western Australia caused by a 2010 trivalent inactivated influenza vaccine. BMJ Open 2011:1:e000016.

11 Gold MS, Effler P, Kelly H, et al. Febrile convulsions after 2010 seasonal trivalent influenza vaccine: implications for vaccine safety surveillance in Australia. Med J Aust 2010;193:492-3.

12 Stokes BP. Ministerial review into the public health response into the adverse events to the seasonal influenza vaccine. government of Western Australia, department of health, 2010. Available: https:// www.health.wa.gov.au/publications/documents/Stokes_Report.pdf

13 Government of Western Australia Department of Health. Western Australian vaccine safety surveillance (WAVSS) system, 2019. Available: https://http://www.healthywa.health.wa.gov.au/Articles/U_ Z/Western-Australian-Vaccine-Safety-Surveillance-system-WAVSS [Accessed $10 \mathrm{Jul}$ 2019].

14 SmartVax. Perth, Au: SmartVax, 2021. Available: http://www. smartvax.com.au [Accessed 31 Mar 2021].

15 AusVaxSafety. National centre for immunisation research and safety. Available: https://www.ausvaxsafety.org.au/ [Accessed 31 Mar 2021]

16 Australian Government Department of Health. Australian immunisation Handbook. Canberra: Australian Technical Advisory Group on Immunisation (ATAGI), 2021. https:// immunisationhandbook.govcms.gov.au

17 Australian Department of Health. AEFI-CAN: a national vaccine safety collaboration. Canberra, AU, 2021. https://http://www.aefican.org.au

18 Cashman P, Macartney K, Khandaker G, et al. Participant-centred active surveillance of adverse events following immunisation: a narrative review. Int Health 2017:9:164-76.

19 Government of Western Australia Department of Health. Pharmacist vaccination code. Available: https://http://www.healthywa.wa.gov. $\mathrm{au} / \sim /$ media/Files/./Pharmacist_vaccination_code.ashx [Accessed 31 Mar 2021].

20 Government of Western Australia Department of Health. Pharmacists - influenza vaccination, 2019. Available: https://ww2.health.wa.gov. au/Articles/S_T/Structured-Administration-and-Supply-Arrangements [Accessed $10 \mathrm{Jul}$ 2019].

21 Australian Government Department of Health. Australian COVID-19 vaccination policy. Available: https://www.health.gov.au/sites/default/ files/documents/2020/11/australian-covid-19-vaccination-policy_1. pdf [Accessed 02 Dec 2020].

22 Centers for Disease Control and Prevention. Ensuring the safety of COVID-19 vaccines in the United States: United States center for diseases control, 2020. Available: https://www.cdc.gov/coronavirus/ 2019-ncov/vaccines/safety.html [Accessed 02 Dec 2020].

23 Nadeem MF, Samanta S, Mustafa F. Is the paradigm of community pharmacy practice expected to shift due to COVID-19? Res Social Adm Pharm 2021;17:2046-8.

24 MedAdvisor, 2020. Available: https://www.medadvisor.com.au/ Home/Landing [Accessed 02 Dec 2020].

25 The University of Western Australia. New program to monitor adverse effects of new vaccines. Available: https://www.uwa.edu.au/news/ Article/2020/March/New-program-to-monitor-adverse-effects-ofnew-vaccines [Accessed 02 Dec 2020].

26 Leeb A, Regan AK, Peters IJ, et al. Using automated text messages to monitor adverse events following immunisation in general practice. Med J Aust 2014;200:416-8.

27 Westphal DW, Williams SA, Leeb A, et al. Continuous active surveillance of adverse events following immunisation using SMS technology. Vaccine 2016;34:3350-5.

28 von Elm E, Altman DG, Egger M, et al. Strengthening the reporting of observational studies in epidemiology (STROBE) statement: guidelines for reporting observational studies. BMJ 2007;335:806-8.

29 Chandler RE. Optimizing safety surveillance for COVID-19 vaccines. Nat Rev Immunol 2020;20:451-2.

30 Kochhar S, Salmon DA. Planning for COVID-19 vaccines safety surveillance. Vaccine 2020;38:6194-8.

31 Vette K, Dalton L, Dey A. Review of pharmacist vaccination reporting to the air final report. National centre for immunisation research and surveillance, 2020. Available: https://ncirs.org.au/sites/default/ files/2020-06/Review\%20of\%20pharmacist\%20vaccination\% 20reporting\%20to\%20the\%20AIR_Final\%20report_May\%202020. pdf [Accessed 02 Dec 2020].

32 Dhanda S, Osborne V, Lynn E, et al. Postmarketing studies: can they provide a safety net for COVID-19 vaccines in the UK? BMJ Evid Based Med 2020. doi:10.1136/bmjebm-2020-111507. [Epub ahead of print: 21 Oct 2020].

33 Wilson SL, Wiysonge C. Social media and vaccine hesitancy. BMJ Glob Health 2020;5:e004206.

34 Blyth CC, Richmond PC, Jacoby P, et al. The impact of pandemic $A(H 1 N 1) p d m 09$ influenza and vaccine-associated adverse events on parental attitudes and influenza vaccine uptake in young children. Vaccine 2014;32:4075-81.

35 Lazarus JV, Ratzan SC, Palayew A, et al. A global survey of potential acceptance of a COVID-19 vaccine. Nat Med 2021;27:225-8.

36 Baroy J, Chung D, Frisch R, et al. The impact of pharmacist immunization programs on adult immunization rates: a systematic review and meta-analysis. J Am Pharm Assoc 2016;56:418-26.

37 Isenor JE, Kervin MS, Halperin DM, et al. Pharmacists as immunizers to improve coverage and provider/recipient satisfaction: a prospective, controlled community embedded study with vaccines with low coverage rates (the improve access study): study summary and anticipated significance. Can Pharm J 2020;153:88-94.

38 Australian Government Ministers Department of Health. Strengthening Australia's immunisation program, 2020. Available: https://www.health.gov.au/ministers/the-hon-greg-hunt-mp/media/ strengthening-australias-immunisation-program [Accessed $04 \mathrm{Dec}$ 2020]. 